NASA Technical Memorandum 89822

\title{
Referencing in Fiber Optic Sensing Systems
}

Grigory Adamovsky

Lewis Research Center

Cleveland, Ohio

(NASA-TH-89822) EEFERENCING IN FIBER OPTIC

SELSING SYSTEAS (AASA) $9 \mathrm{~F}$ CSCL $20 \mathrm{~F}$

N87-20475

G3/33 Unclas

Prepared for the

Technical Symposium of Optics, Electro-Optics, and Sensors sponsored by the Society of Photo-Optical Instrumentation Engineers Orlando, Florida, May 17-22, 1987 
Referencing in fiber optic sensing systems

Grigory Adamovsky

National Aeronautics and Space Administration, Lewis Research Center, Mail Stop 77-1, 21000 Brookpark Road, Cleveland, Ohio 44135

\section{Abstract}

Different techniques to account for losses induced by the environment on signals in intensity modulation fiber optic sensing systems are described and analyzed.

\section{Introduction}

Intensity modulating optical transducers are attractive for use in sensing systems due to their relative simplicity and low cost. A fiber-optic link is often used to channel information about the measurand from a sensor-transducer to remotely located transmittingreceiving electronics. External disturbances affect the signal propagating along the link and induce variable transmission losses in the system. The susceptibility of the sensing system to the disturbances acting on the fiber-optic link increases noise and often makes the measurements ambiguous.

Connectors and splices cause additional losses in the transmission 1 ine. These losses may change every time the connector is remated, or a new splice is made. Thus recalibration is needed after such operations. This procedure can take too much time, require special equipment, and specially trained personnel.

In order to compensate for intensity variations in the fiber-optic 1 ink, connectors, and light source a system with two channels, signal and reference, has to be constructed. The most commonly used methods to build such a system involve either spatial, frequency (wavelength), or temporal separation of the channels. (See Figure l.)

This paper discusses different ways to implement the separation of the signal and reference channels.

\section{Spatial separation of channels}

To separate the signal and reference channels in the space domain two fibers made of the same material and of the same length are used. Schematics of a sensing system with two physically separated channels is shown in Fiqure 1 (a). One fiber connects the source, sensor, and photodetector and forms a signal channel. Another fiber that is placed alongside the first one but bypasses the sensor-transducer makes up the reference channel. Because both fibers are subjected to the same external conditions and experience the same variations in the light source intensity, these effects can be easily factored out. This technique is simple to implement, does not require complex and expensive electronics, and works with almost all types of intensity sensors.

Fiber optic sensing systems employing the described technique have been built to measure temperature, displacement, and pressure.1,2

One of the disadvantages of this technique is the use of two fibers. The presence of two physically similar channels requires two equivalent sets of optical and electronic components. This decreases the reliability of the sensing system. In addition the fibers that form the channels may not be exactly the same, and thus the effect of the environment on the signals propagating along the fibers may also be different. The use of dual core fiber may minimize this problem and make the spatial separation of channels more attractive. Unfortunately technological problems in manufacturing the dual core fibers have to be overcome.

\section{Separation in wavelength domain}

In this method a dual wavelength technique is used to make up the reference and signal channels, and the same fiber link can be used for both channels. one of the wavelengths is sensitive to disturbances that occur at the sensor-transducer. This wavelength forms the signal channel. The second wavelength makes up the reference channel and lies outside the bandwidth of spectral sensitivity of the sensor, and the signal at this wavelength is not affected by the measurand. Upon reaching the photodetectors this siqnal is evaluated at the wavelengths used, and thus the information about the measurand is retrieved. 
A schematic of a sensing system with wavelength separation of channels is shown in Figure $1(b)$.

Sensing systems with wavelength separated channels have been reported that measure temperature, pressure, and displacement. $3-6$

In order to prevent crosstalk between the reference and signal channels in the twowavelength scheme, generally, two light sources with their central wavelengths well sepa$r$ ated and two photodetectors are needed. Optical filters in front of either the sources or the detectors tune the sensing system to particular wavelengths and increase the sensitivity of the system to the measured parameter.

The utilization of two light sources and two photodetectors decreases reliability of the system and requires identical behavior for the transmission and reception optics and electronics at both optical frequencies. At the same time the optical filters greatly reduce the power budget of the system.

A configuration discussed in detail in Reference 7 uses fluorescent properties of some materials. Because the intensity of the fluorescence in some of these materials has very weak temperature dependence over the temperature range 300 to $500 \mathrm{~K}^{8}$ it is possible to use a fluorescence band as reference, and the emission spectrum of the source as the signal channel. Thermal stability of the fluorescence spectrum, sufficient separation of the wavelengths, and availability of high power LEDs make it possible to build a temperature sensor based on this principle.

A fiber optic thermometer with a fluorescence reference channel has been demonstrated.9 The main advantage of this approach lies in the use of just one light source.

Due to the nature of physical phenomena employed in the two-wavelength approach the spectral distribution of the light source intensity and its thermal stability play an important role in the system performance. The graphs presented in Figure 2 show the computed power that reaches the photodetector after passing through a piece of absorbing material as a function of temperature for different sources. The sensing mechanism in the model was chosen to be the temperature dependent absorption in neodymium, and the computations covered the spectral region from 700 to $900 \mathrm{~nm}$. As a hypothetical sample, a $10 \mathrm{~mm}$ thick piece of laser glass with 8 wt $8 \mathrm{Nd}_{2} \mathrm{O}_{3}$ was used, and this material's physical parameters were used in the calculations. In these computations the optical sources were assumed to be of unity power but with the central wavelength of 839,840, and $841 \mathrm{~nm}$. The spectral bandwidth of $30 \mathrm{~nm}$ was assumed to be the same for all three sources. This simulated shift of $1 \mathrm{~nm}$ in the spectral emission in a real environment could be generated by a change in the semiconductor light source junction temperature. The corresponding change in the junction temperature that causes such a shift could be just several degrees depending on the semiconductor material. Thus thermocooled light sources may be necessary in order to achieve the required stability.

\section{Time domain referencing}

In this type of referencing the channels are separated temporally over the same fiber optic link. Different schemes to construct a reference channel in the time domain have been described elsewhere.10 The principal element of these schemes is a fiber optic (FO) loop. A light pulse of a short duration launched into such a loop results in a train of pulses of different amplitudes. The information about the measurand is retrieved by comparing pulses in the same train. This technique can be used with different sensorstransducers and has been demonstrated to determine pulse spreadingli and birefrintransducers and has been demonstrated to determine pulse spread
gence, 12 as well as to measure pressure 13 and temperature. 14

A schematic of a reflective type Fabry-Perot Fo loop with an incorporated sensor is shown in Figure 1 (c). The Fabry-Perot Fo. loop is formed by a semitransmittingsemireflecting mirror 1, a reflecting mirror 2 which is a part of the sensor, and a piece of fiber between the mirrors long enough to generate a train of pulses.

The main disadvantage of this technique is the fact that the entire Fo 1oop is sensitive to disturbances. This makes it difficult to determine whether the signal change is due to the measurand or to the entire Fo loop. To avoid ambiguity in measurements the loop should be made as short as possible. However, the shorter loop requires an initial pulse of shorter duration to generate a pulse train.

To process pulses of short duration high speed oscilloscopes or streak camerasl 5 are required. High-speed oscilloscopes permit the processing of high bandwidth ( 1 l GHz) analog signals. This corresponds to a FO loop length of $0.2 \mathrm{~m}$ or shorter (as suming that the fiber used in the Fo loop has the index of refraction of 1.5). Potentially, a streak 
camera can resolve an analog signal with a bandwidth up to $10 \mathrm{GHz}$ and above; and high-speed fiber-pigtailed diodes and optical receivers with modulation bandwidths up to $10 \mathrm{GHz}$ are also now available. 16

With this technology employed, the limitation on the system is attributed to the transmission bandwidth of the fiber optic link. This bandwidth depends on the length of the fiber cable between the sensor-transducer and processing electronics and the type of fiber used. For instance, a $100 \mathrm{~m}$ long $50 \mathrm{\mu m}$ core diameter graded index fiber with profile optimized for $1 \mathrm{GHz} \mathrm{km}$ bandwidth can support an analog signal with $10 \mathrm{GHz}$ bandwidth.

However, despite the ability to process high bandwidth analog signals, the technique using streak cameras has limited applications due to large size and weight of the equipment. In order to make time domain referencing a valuable technique for sensor applications where size and weight play a considerable role special signal processing methods have to be developed.

\section{Combination techniques}

In some cases none of the three basic referencing techniques analyzed here provide good compensation for losses and at the same time satisfy the requirements for low cost and maintainability.

Improved performance of a sensing system could be achieved by combining two of the referencing techniques. Figure $3(a)$ shows a schematic of a sensing system with a combination of spatial and temporal referencing. The reference channel is constructed in the space domain by using two fibers in the sensor head, one of which is a dumm; and in the time domain by using a delay line $L$ in the dummy channel. The system has the advantage of only one fiber link between the sensor head and the electronics, but suffers from ambiguities in determining the effect of disturbances on the signal and dummy fibers in the sensor head. A compensated displacement sensor utilizing such a technique is described in Reference 17 .

Another referencing technique involving time and wavelength domain referencing has been reported in Reference 18. Two wavelengths represent the signal and reference channels in the wavelength domain. One of the wavelengths is affected by the measurand and the other wavelength is not. The information about the measurand is retrieved by comparing the backscattered signals at both wavelengths from points A and B (see Figure 3 (b)) which 1 ie before and after the sensor. The referencing in the time domain is provided by pulsing the sources and proper timing of detectors and the backscattered signals. Despite some additional stability gained by utilizing this type of combined referencing the system still has problems associated with the use of two wavelengths.

\section{Concluding remarks}

The difference referencing techniques described in this paper illustrate how losses resulting from the transmission link and changes in the optical source intensity can be factored out of intensity modulation sensing systems. The choice of technique is based on the parameter to be measured, sensor configuration, and stability requirements.

\section{References}

1. A.M. Scheggi, M. Brenci, G. Conforti, R. Falciai, and G.P. Preti, "Optical Fiber Thermometer for Medical Use," in Optical Fibre Sensors, IEE CP-221, pp. 13-16 (1983).

2. J.W. Berthold, and L.A. Jeffers, "Fiber Optic Method for Sensing Diaphragm Deflection," in Fiber Optic and Laser Sensors, E.L. Moore and O.G. Ramer, eds., Proc. SPIE 412, pp. 90-95(1983).

3. E. Theocharous, "Displacement and Temperature Multimeasurant Transducer," in Second International Conference on Optical Fiber Sensors, R.T. Kersten and R. Kist, eds., PrOC. SPIE 514, pp. 199-203 (1984).

4. B.E. Jones and R.C. Spooncer, "An Optical Fiber Pressure Sensor Using a Holographic Shutter Modulator with Two-wavelength Intensity Referencing," in Second International Conference on Optical Fiber Sensors, R.T. Kersten and R. Kist, eds., Proc. SPIE 514 , pp. 223-226 (1984) .

5. E. Snitzer, W.W. Morey, and W.H. Glenn, "Fiber Optic Rare Earth Temperature Sensors," in Optical Fibre Sensors, IEE CP-221, pp. 79-81 (1983). 
6. K. Kyuma, S. Tai, T. Sawada, and M. Nunoshita, "Fiber-optic Instrument for Temperature Measurement," IEEE J. Quantum Electron., Vol. QE-18, pp. 676-679 (1982).

7. K.T.V. Grattan and A.W. Palmer, "Fluorescence Monitoring for optical Temperature Sensing," in Fiber Optics '86, Proc. SPIE 630, pp. 256-265 (1986).

8. J.R. Thornton, W.D. Fountain, G.W. Flint, and T.G. Crow, "Properties of Neodymium Laser Materials," Appl. Opt., Vol. 8, pp. 1087-1102 (1969).

9. K.T.V. Grattan, R.K. Selli, and A.W. Palmer, "Fiber-Optic Absorption Temperature Sensor Using Fluorescence Reference Channel," Rev. Sci. Instrum., Vol. 57, pp. $1175-1178$ (1986).

10. G. Adamovsky, "Time Domain Referencing in Intensity Modulation Fiber Optic Sensing Systems," in Optical Testing and Metrology, C.P. Grover, ed., Proc. SPIE 66l, pp. $145-151\left(\frac{1986)}{1}\right.$.

11. L.G. Cohen, "Shuttle Pulse Measurements of Pulse Spreading in an Optical Fiber," Appl. Opt., Vol. 14, pp. $1351-1356$ (1975).

12. C.S. Brown and F.T. Stone, "Birefringence Measurements of Single-Mode Fibers Using the Polarization Shuttle Pulse Technique," J. Lightwave Technol., Vol. LT-4, pp. 30-33 $(1986)$.

13. G. Edamovsky, "All-Fibre Sensing Loop Using Pulse-Modulated Light-Emitting Diode," Electron. Lett., Vo1. 21, pp. $922-923$ (1985).

14. G. Adamovsky and N.D. Piltch, "Fiber-Optic Thermometer Using Temperature Dependent Absorption, Broadband Detection, and Time Domain Referencing," Appl. Opt., Vol. 25 pp. 4439-4443(1986).

15. J. Chang, W. Filter, G. Lockwood, and B. Neyer, "A Multi-GHz Multi-Channel Photonic Analog Data-Processing System," in Fiber Optics and Laser Sensors II, E.L. Moore and O.G. Ramer, eds., Proc. SPIE 478 , pp. 80-85 (1984).

16. K.Y. Lau and A. Yariv, "Ultra-High Speed Semiconductor Lasers," IEEE J. Quantum Electron., Vol. QE-21, pp. 121-138 (1985).

17. D.E.N. Davis, J. Chaimowicz, G. Economou, and J. Foley, "Displacement Sensor Using a Compensated Fiber Link," in second International Conference on optical Fiber Sensors, R.T. Kersten and R. Kist, eds., Proc. SPIE 514, pp. 387-390 (1984).

18. E. Theocharous, "Differential Absorption Distributed Thermometer," in Optical Fibre Sensors, IEE CP-221, pp. 10-12 (1983). 


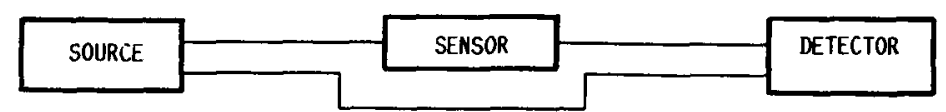

(A) SPATIAL SEPARATION OF CHANNELS.

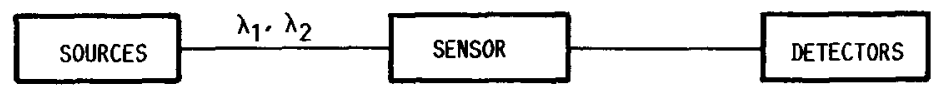

(B) SEPARATION OF CHANMELS IN WAVELENGTH DOMAIM.

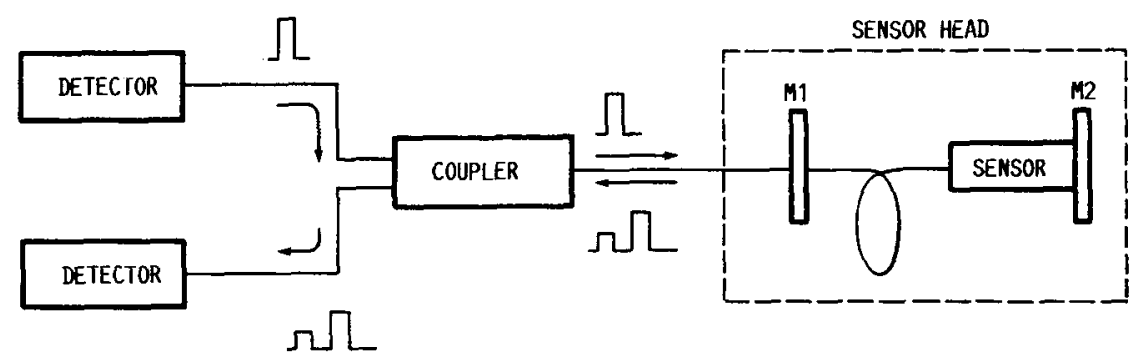

(C) TEMPORAL SEPARATION OF CHANNELS.

FIGURE 1. - SCHEMATIC DIAGRAMS OF THREE BASIC REFERENCING TECHNIQUES. 


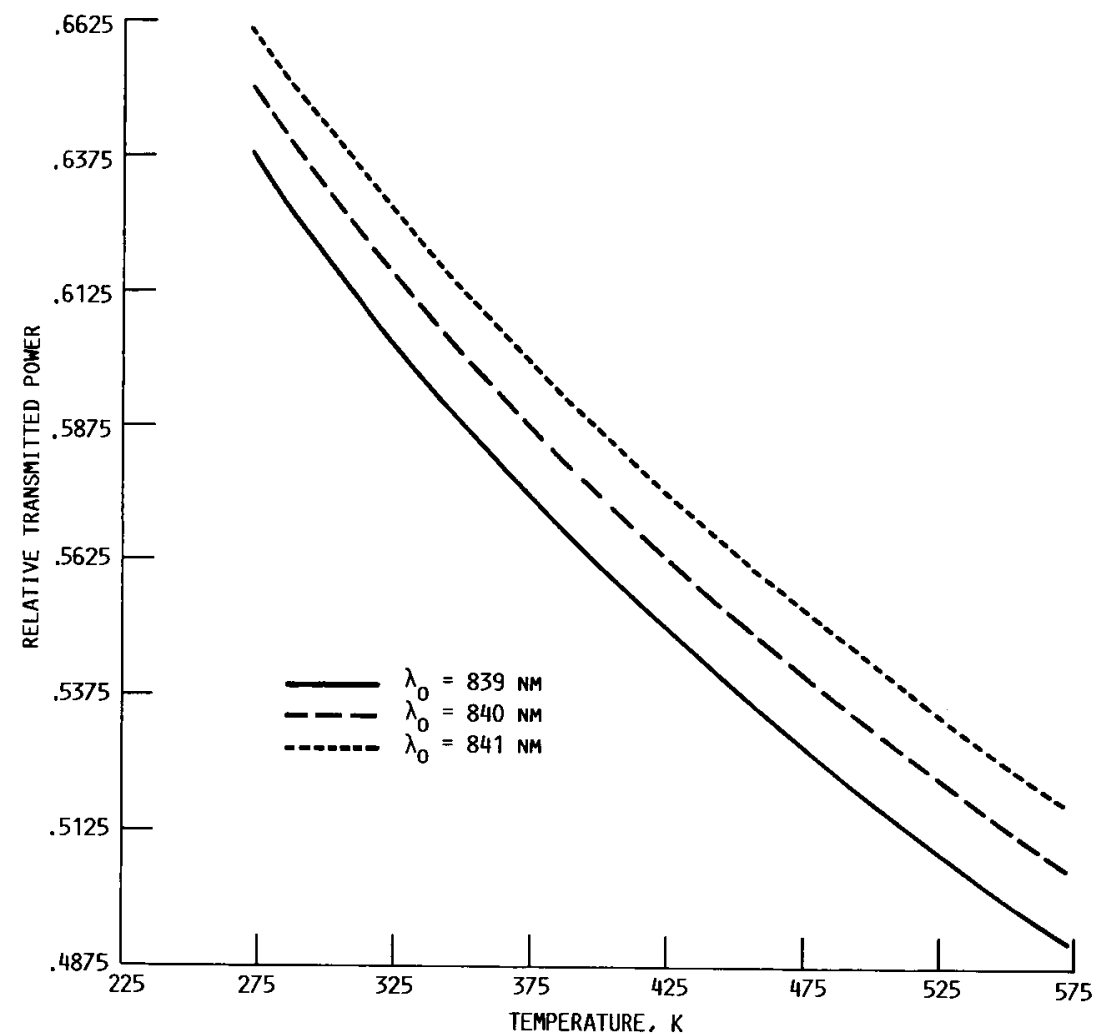

FIGURE 2. - CALCULATEd RELATIVE POWER FROM SOURCES WITH THE SAME SPECTRAL BANDWIDTH OF $30 \mathrm{NM}$ AND DIFFERENT CENTRAL HAVELENGTH $\lambda_{0}$, TRANSMITTED THROUGH A 10 MM THICK SAMPLE OF GLASS DOPED WITH 8 WEIGHT PERCENT Nd $\mathrm{O}_{3}$ AS FUNCTION
OF TEMPERATURE. 


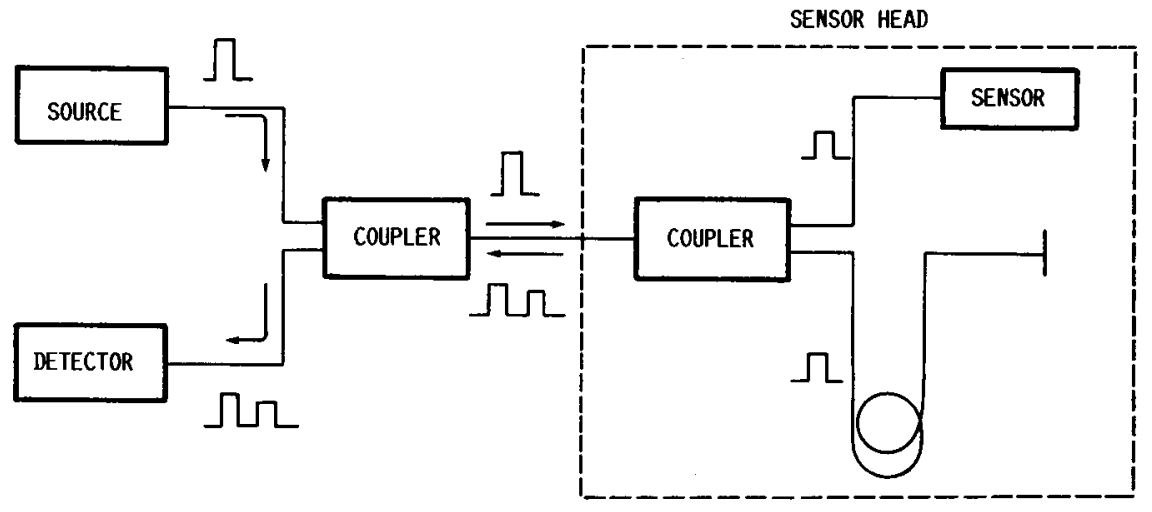

(A) SYSTEM WITH SIMULTANIOUS SPATIAL AND TEMPORAL REFERENCING.

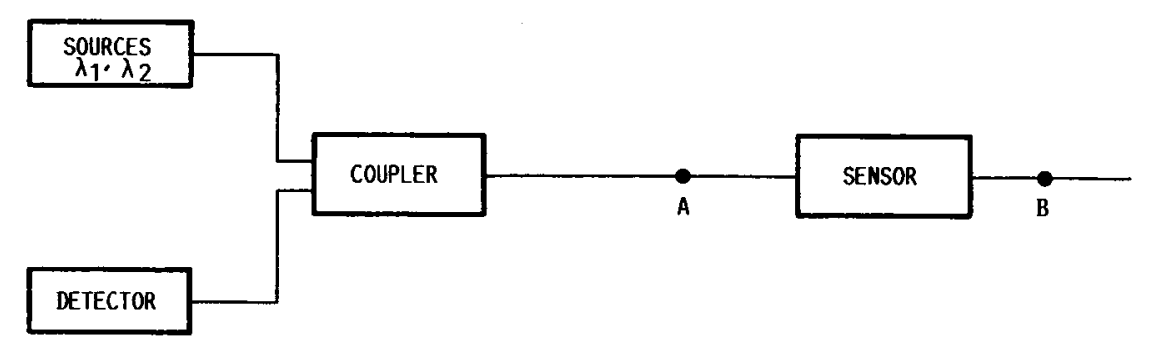

(B) REFERENCING IN TIME AND WAVELENGTH DOMAINS.

FIGURE 3. - DIAGRAMS OF COMBINAIION REFERENCING TECHNIQUES. 


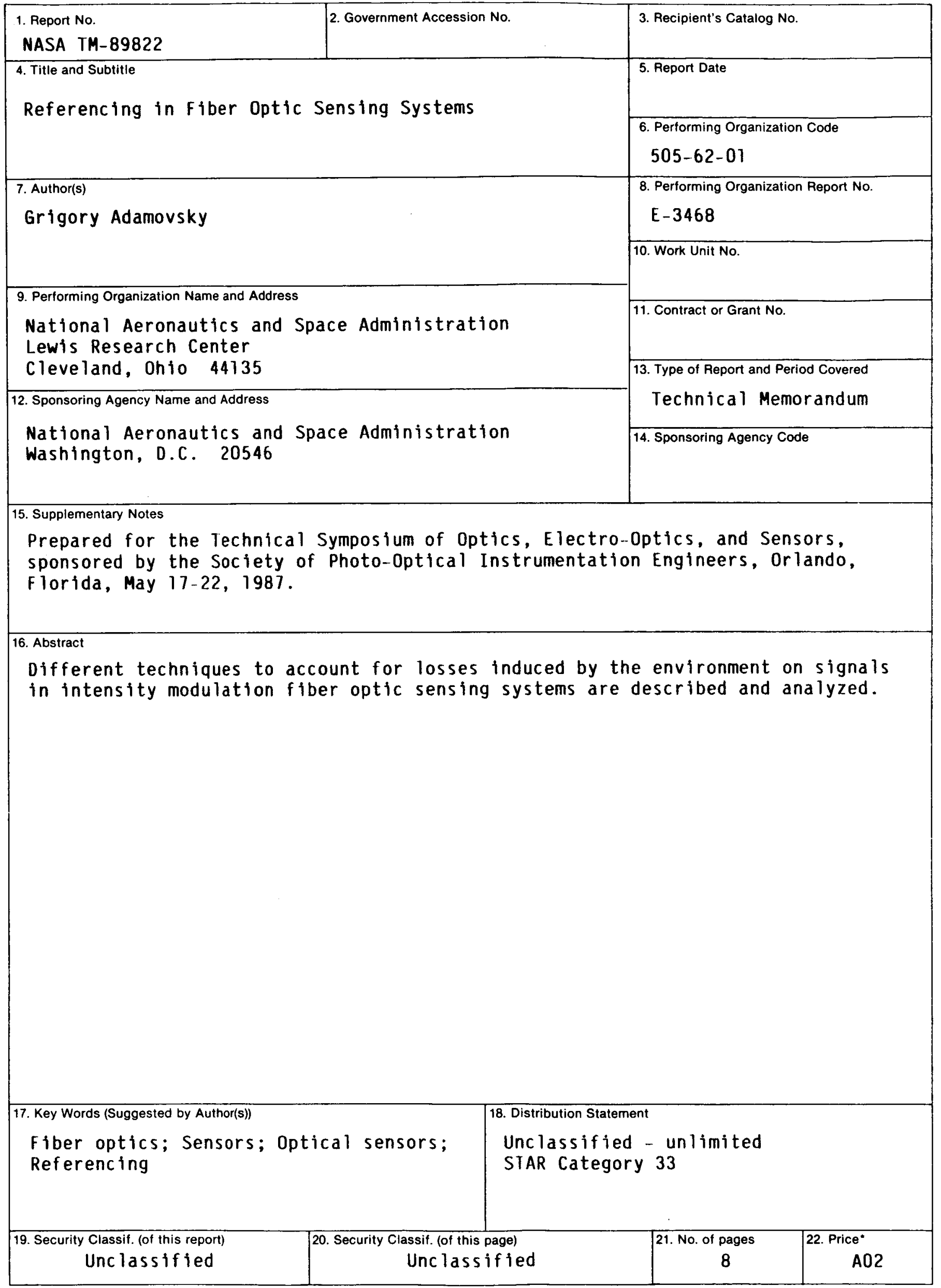

*For sale by the National Technica! Information Service, Springfield, Virginia 22161 